\title{
Transcript Profiling in the Barley Mildew Pathogen Blumeria graminis by Serial Analysis of Gene Expression (SAGE)
}

\author{
Stephen W. Thomas, ${ }^{1,3}$ Mikkel A. Glaring, ${ }^{1,2}$ Søren W. Rasmussen, ${ }^{1,2}$ Julia T. Kinane, ${ }^{1}$ and Richard P. \\ Oliver $^{3}$ \\ ${ }^{1}$ Department of Physiology, Carlsberg Laboratory, Gamle Carlsberg Vej 10, Copenhagen Valby, DK-2500, Denmark; \\ ${ }^{2}$ Department of Yeast Genetics, Carlsberg Laboratory, Gamle Carlsberg Vej 10, Copenhagen Valby, DK-2500, Denmark; \\ ${ }^{3}$ Australian Centre for Necrotrophic Fungal Pathogens, Division of Science and Engineering, Murdoch University, \\ WA 6150, Australia
}

Submitted 29 October 2001. Accepted 13 April 2002.

\begin{abstract}
The fungal pathogen Blumeria graminis f. sp. hordei develops on the barley leaf via distinct, morphologically well-defined stages. After landing on a host plant, the conidia rapidly germinate to form a primary germ tube. Subsequently, an appressorial germ tube emerges from the conidium and differentiates an appressorium from which penetration of the host cell wall is attempted. We have used serial analysis of gene expression to provide a measurement of messenger RNA contents in ungerminated conidia, during conidial germination, and during appressorium formation. The resulting data provide a resource for the characterization of changes in transcript accumulation during early development of $B$. graminis.
\end{abstract}

Filamentous fungi impact numerous aspects of human activities. Some species provide enzymes for industrial use and food preparation. Others cause devastating diseases of agricultural crops, animals. and humans. Some produce antibiotics, or serve as model organisms for scientific research. Therefore, it is surprising that large-scale characterization of mRNA levels in filamentous fungi has not been performed. The present analysis depicts the characterization of mRNA abundance of individual genes during early development of the obligate, filamentous fungal plant pathogen Blumeria (syn. Erysiphe) graminis (DC.) Speer f. sp. hordei, the causal agent of barley powdery mildew.

Much of the current knowledge regarding events surrounding conidial germination and appressorium differentiation comes from studies of facultative plant pathogens. Analysis of gene deletion mutants has elucidated the roles of several genes and environmental factors in early development (Dean 1997; Hamer and Talbot 1998; Howard and Valent 1996; Mendgen et al. 1996; Xu 2000). Large-scale transcription profiling during conidial germination and appressorium formation are, however, entirely lacking.

Barley powdery mildew is caused by $B$. graminis, an obligate pathogen requiring the host for completion of its life cycle. Upon contact with a solid surface, the conidia produce a primary germ tube, whose role appears to be to sense the suitability of the surface for further fungal development (Kinane et al. 2000). On inductive surfaces, an appressorial germ tube

Corresponding author: R. P. Oliver, Telephone: +61 (8) 9360 7404; Fax: +61 (8) 9360 6303; E-mail: roliver@central.murdoch.edu.au. emerges that subsequently differentiates into the functional appressorium from which penetration of the underlying host cell is attempted. The characterization of gene expression and function in obligate pathogens is difficult due not only to an inability to recover nonpathogenic mutants, but also because the isolation of suitably defined material is problematic. Fortunately, some cellulose membranes support the efficient formation of $B$. graminis appressoria (Kinane et al. 2000; Kobayashi et al. 1991), allowing the preparation of suitable experimental material in the absence of contaminating plant tissue. Appressoria formed in vitro are known to be functional through the observation of penetration pores in cellulose membranes directly below the appressorial structures (Kobayashi et al. 1991). We have measured transcript abundance during $B$. graminis germination and appressorium development on inductive cellulose membranes using the serial analysis of gene expression (SAGE) procedure (Velculescu et al. 1995). Here, we analyze approximately 60,000 tags isolated from ungerminated conidia, germinating conidia, and appressoria of B. graminis. This work represents the first large-scale quantification of individual transcripts during development of a pathogenic fungus.

\section{RESULTS}

Analysis of gene number and expression during development.

Sequencing of approximately 20,000 tags from each of three SAGE libraries, representing ungerminated conidia, germinating conidia with a primary germ tube, and conidia with developing appressoria, identified 18,691 different 14-base tags. Ambiguous tags that were present only once were removed from the analysis as described (Lash et al. 2000). Seven tags that may have been derived from mispriming of residual ribosomal RNA were also removed from the analysis. This process identified 6,336 different tags that could be assumed to represent unique transcripts.

Statistical analyses of the differences in the number of specific tags in the three populations were performed and tags whose frequency changed significantly at the $95 \%$ confidence level between the three developmental stages were enumerated (Table 1). The determination of statistically robust (confidence interval 95\%) alteration in specific tag frequencies, given that a total of approximately 20,000 tags were sequenced in each of three populations, requires that the representative tag must be identified a minimum of six times in at least one of the popula- 
tions studied. A total of 916 tags met the above criteria. In all, 463,563 , and 524 tags were present in the ungerminated conidia, germinated conidia, and appressorial populations, respectively, in six or more copies, indicating that many transcripts were abundant in more than one of the developmental stages under investigation. Totals of 106 and 94 tags displayed significantly increased frequency during germination and appressorial development, respectively, (Table 1.). A similar number of tags displayed a significant reduction in frequency during germination and appressorium development (82 and 105 , respectively). It is important to note that the statistical analysis of the data allows inferences to be made only about the significance of observed differences in tag number from one population to another. Hence, the statistical analysis of a tag with a count of five tags in ungerminated conidia, one tag in germinated conidia, and eight tags in appressoria allows the conclusion of a significant increase in the transition from germination to appressorial development. It does not allow an inference to be made about the relative frequency of occurrence in ungerminated conidia, only that it is not significantly different from the level in germinated conidia or appressoria.

\section{Mapping of tags to genes.}

Comparison of the SAGE tags with the available expressed sequence tag (EST) sequences for B. graminis (Thomas et al. 2001) has allowed the assignment of 1,274 tags (20\%) to sequenced EST clones. Of the 1,669 unique $B$. graminis ESTs, $80 \%$ of the representative tags have been detected in the SAGE analysis. Clearly, genes with abundant transcripts are more likely to be represented in both SAGE profiles and EST databases.

The 20 most frequently sequenced tags from each of the developmental stages studied and their corresponding EST clone are presented in Table 2. Many of the genes identified have high levels of mRNA present in all three stages studied, suggestive of an ongoing requirement for these gene products during development.

\section{Statistical analysis of differential gene expression.}

A total of 394 different tags displayed a significant change in the frequency of occurrence during the transition from the ungerminated conidium into an appressorial infection structure. The individual tags that have linked ESTs are listed in Table 3. This analysis identifies 183 genes displaying a flux in mRNA quantity between at least two of the stages analyzed. Blast analysis (Altschul et al. 1990, 1997; Thomas et al. 2001) identified putative homologues of 126 of these genes in other organisms; 57 genes have no previously characterized homologue.

\section{Comparison to known expression profiles.}

Prior to this study, there had been few attempts to measure the expression of individual genes during development of $B$. graminis. The quantification of transcripts of two chitin synthase genes, $B g C h s 1$ and $B g C h s 2$, has been determined by semiquantitative reverse transcription polymerase chain reaction (RT-PCR), which indicated that $B g C h s 1$ mRNA accumulated during germination and decreased slightly during appres-

Table 1. Numbers of tags displaying significant changes in transcript abundance during germination and development of the appressorium ${ }^{\mathrm{a}}$

\begin{tabular}{lcc}
\hline $\begin{array}{l}\text { Transcriptional } \\
\text { regulation }\end{array}$ & $\begin{array}{c}\text { Ungerminated } \rightarrow \\
\text { germinated conidia }\end{array}$ & $\begin{array}{c}\text { Germinated conidia } \\
\rightarrow \text { appressoria }\end{array}$ \\
\hline $\mathrm{Up}$ & $106(57)$ & $94(46)$ \\
Down & $82(34)$ & $105(51)$ \\
\hline
\end{tabular}

${ }^{\mathrm{a}}$ Numbers in parentheses are tags with corresponding genes in the Blumeria expressed sequence tag database (Thomas et al. 2001). sorium formation (Zhang et al. 2000). BgChs2 had a similar profile but at a much lower transcript abundance. The occurrence of the tag for $B g C h s 1$ in the SAGE profiles, albeit in low numbers (ungerminated, three tags; germinated, nine; appressoria, four), and the absence of a tag for $B g C h s 2$ are entirely consistent with the published transcript analysis (Zhang et al. 2000). RT-PCR is evidently a more sensitive technique than SAGE (on 20,000 tags) for the detection of mRNA of individual genes.

Northern analysis data is available for glyceraldehyde 3phosphate dehydrogenase $(G p d), g E g h 7$, and $g E g h 16$ (Justesen et al. 1996). $g E g h 7$ originally was reported to be a single copy gene, although we have since identified one other homologue, $g E g h 7.2$ (Thomas et al. 2001). $g E g h 16$ represents a small gene family comprising $g E g h 16, g E g h 16.2$, and $g E g h 16.3$. Furthermore, the original study utilized spores germinated on glass pates, a noninductive surface. Insofar as the data are comparable, the trends in the SAGE data are consistent with the earlier Northern data. These results highlight the facility of SAGE to separate transcript profiles of individual members of gene families. Transcripts of $g$ Eghl6 homologues recently have been shown to be abundant in appressoria of Magnaporthe grisea (Kamakura et al. 1999), suggesting that gEgh16 has a role in appressorium maturation.

\section{DISCUSSION}

Array-based hybridization (Schena et al. 1995) and SAGE (Velculescu et al. 1995) represent the main techniques for high-throughput transcriptional profiling. Both techniques correlate well in terms of determining absolute levels of specific mRNAs as well as the identification of differential mRNA accumulation in different materials (Ishii et al. 2000). We chose the SAGE technique because it can measure the abundance of all transcripts with precision and because SAGE data is generated in a reusable, digital format from uncharacterized genes and is thus particularly suited to nonmodel systems and to collaborative studies. The data is viewed as a resource, allowing current and future researchers to rapidly assess particular transcript abundance during early development of newly identified genes arising from both EST and genome sequencing data. This is particularly important in light of continued sequencing of ESTs during development of the mildew colony and the ongoing plans for genome sequencing of $B$. graminis. (Eckert et al. 2001; M. Glaring, unpublished data). Thus far, we have analyzed the data against an EST database currently comprising 1,669 unigene sequences (Thomas et al. 2001). This preliminary analysis identifies some of the genes having changes in mRNA content during development and therefore gives an insight into some of the processes that may be important during infection structure formation. While $20 \%$ of tags are identified in the current EST analysis, ongoing sequencing efforts will undoubtedly allow more identifications to be made. In addition, the genes corresponding to unknown tags with interesting patterns of transcript accumulation may be rapidly isolated using one of the current PCR methods of tag-to-gene cloning (Polyak et al. 1997; Yu et al. 1999).

\section{Identification of genes from SAGE tags.}

Of the 1,669 EST sequences that are available, 1,274 were identified as SAGE tags. Identification of the most sequenced tags from each developmental stage (Table 2) showed that the majority displayed high transcript abundance in all three stages examined. The most sequenced tag was representative of a putative metallothionein, a protein associated with oxidative and heavy metal stress (Andrews 2000). Metallothionein homologues have been identified as specifically expressed 
during appressorium formation in Colletotrichum gloeosporioides (Hwang and Kolattukudy 1995). The presence of a heat-shock protein and a cytochrome p450 gene support the view that stress responses are fully activated during development. Other genes with considerable transcript levels, as judged by SAGE, are involved in protein synthesis, modifica- tion, and degradation that may indicate that early germling development involves rapid protein synthesis and degradation (McCafferty and Talbot 1998).

Of the 1,274 genes with linked ESTs, 183 had significant changes in transcript abundance during development. These genes have been divided among 10 classes representing different

Table 2. Identification of the twenty genes with the highest transcript levels in each of the developmental stages studied ${ }^{\mathrm{a}}$

\begin{tabular}{|c|c|c|c|c|c|}
\hline Tag & $\begin{array}{c}\text { GenBank } \\
\text { accession no. }\end{array}$ & Putative homologue & $\mathbf{U g}$ & Ge & Ap \\
\hline \multicolumn{6}{|c|}{ Ungerminated conidia } \\
\hline AAGGGAGGAT & AW791711 & Metallothionein & 286 & 209 & 501 \\
\hline CCGCAGGGCT & AW790864 & Subtilisin-like serine protease & 159 & 135 & 212 \\
\hline GCTAACGCAG & AW791080 & Peptidyl-prolyl cis-trans isomerase & 158 & 143 & 217 \\
\hline TCACCCCACT & AW791647 & Novel EST (D00719) & 156 & 88 & 129 \\
\hline AATTAGCTAA & AW791518 & Glucose-repressible gene & 113 & 70 & 46 \\
\hline TCAATTCTCT & AW791559 & Elongation factor $1-\alpha$ & 111 & 117 & 115 \\
\hline TGTCATAGCT & AW790559 & Retrotransposon & 95 & 144 & 86 \\
\hline TCGAAGGGTG & AW788687 & GEL1 protein & 70 & 49 & 75 \\
\hline ATACCAAAAT & AW791955 & 30-kDa heat shock protein & 63 & 10 & 3 \\
\hline GCTGCAACAG & $\ldots$ & Unknown & 61 & 0 & 0 \\
\hline GAGGTCGGGC & AW791580 & Male sterility 2-like protein & 58 & 86 & 112 \\
\hline GCACTATTGA & AW788796 & Mycosphaerella graminicola EST AW181057 & 56 & 91 & 141 \\
\hline TTAGTCTGTT & AW792295 & Novel EST (D01117) & 55 & 16 & 14 \\
\hline CTTCGATGGT & AW791136 & Mannosyl-oligosaccharide $\alpha$-1,2-mannosidase & 55 & 25 & 86 \\
\hline CGAGCGGTGA & AW791967 & Ubiquitin conjugating enzyme E2-16 kDa & 53 & 77 & 67 \\
\hline ATGGGTGGAC & AW792426 & Related to chitinase 3 & 52 & 45 & 90 \\
\hline GGGTTGCAAA & AW792221 & Aspergillus nidulans EST AI211830 & 51 & 15 & 16 \\
\hline TCCCTATTAA & $\ldots$ & Unknown & 49 & 12 & 6 \\
\hline GAGCGCGTGA & AW790920 & Isotrichodermin C15 hydroxylase & 49 & 13 & 36 \\
\hline CGTGGGGATA & AW791719 & Cytochrome p450-like TBP & 49 & 63 & 70 \\
\hline \multicolumn{6}{|l|}{ Germinated conidia } \\
\hline AAGGGAGGAT & AW791711 & Metallothionein & 286 & 209 & 501 \\
\hline TGTCATAGCT & AW790559 & Retrotransposon & 95 & 144 & 86 \\
\hline GCTAACGCAG & AW791080 & Peptidyl-prolyl cis-trans isomerase & 158 & 143 & 217 \\
\hline CCGCAGGGCT & AW790864 & Subtilisin-like serine protease & 159 & 135 & 212 \\
\hline TTTCAATCTG & AW790713 & Lipase 2 precursor & 32 & 134 & 135 \\
\hline AAAAAAAAAA & $\ldots$ & Unidentifiable & 41 & 131 & 21 \\
\hline TCAATTCTCT & AW791559 & Elongation factor $1-\alpha$ & 111 & 117 & 115 \\
\hline GCACTATTGA & AW788796 & Mycosphaerella graminicola EST AW181057 & 56 & 91 & 141 \\
\hline TCACCCCACT & AW791647 & Novel EST (D00719) & 156 & 88 & 129 \\
\hline GAGGTCGGGC & AW791580 & Male sterility 2-like protein & 58 & 86 & 112 \\
\hline CAGAGAGGCA & AW789769 & Novel EST (C01328) & 44 & 85 & 41 \\
\hline GTGAAATCCG & AW790071 & $\operatorname{gEgh} 7.2$ & 27 & 80 & 84 \\
\hline CGAGCGGTGA & AW791967 & Ubiquitin conjugating enzyme E2-16 kDa & 53 & 77 & 67 \\
\hline GGTTTCGGGC & AW788286 & 60s ribosomal protein $\mathrm{p} 2$ & 46 & 74 & 52 \\
\hline ACACCTAGAT & AW789656 & Enolase & 39 & 73 & 13 \\
\hline AATTAGCTAA & AW791518 & Glucose-repressible gene & 113 & 70 & 46 \\
\hline TCCTACATAG & AW787954 & Retrotransposon & 23 & 67 & 32 \\
\hline CGTGGGGATA & AW791719 & Cytochrome p450-like TBP & 49 & 63 & 70 \\
\hline ATATAGGCGG & AW791010 & Novel EST (D00348) & 10 & 50 & 31 \\
\hline TCGAAGGGTG & AW788687 & GEL1 protein & 70 & 49 & 75 \\
\hline \multicolumn{6}{|l|}{ Appressoria } \\
\hline AAGGGAGGAT & AW791711 & Metallothionein & 286 & 209 & 501 \\
\hline GCTAACGCAG & AW791080 & Peptidyl-prolyl cis-trans isomerase & 158 & 143 & 217 \\
\hline CCGCAGGGCT & AW790864 & Subtilisin-like serine protease & 159 & 135 & 212 \\
\hline CGCGCAATGA & AW788949 & Neurospora crassa EST AI397742 & 16 & 27 & 149 \\
\hline TGTGTGCGCG & AW791142 & Myb-like transcription factor & 14 & 27 & 142 \\
\hline GCACTATTGA & AW788796 & Mycosphaerella graminicola EST AW181057 & 56 & 91 & 141 \\
\hline TTTCAATCTG & AW790713 & Lipase 2 precursor & 32 & 134 & 135 \\
\hline TCACCCCACT & AW791647 & Novel EST (D00719) & 156 & 88 & 129 \\
\hline TCAATTCTCT & AW791559 & Elongation factor $1-\alpha$ & 111 & 117 & 115 \\
\hline GAGGTCGGGC & AW791580 & Male sterility 2-like protein & 58 & 86 & 112 \\
\hline GGCCCATAGG & AW789547 & Novel EST (C01191) & 34 & 34 & 91 \\
\hline ATGGGTGGAC & AW792426 & Related to chitinase 3 & 52 & 45 & 90 \\
\hline CTCGGCCCAG & AW791162 & Hex 1 & 12 & 44 & 89 \\
\hline TGTCATAGCT & AW790559 & Retrotransposon & 95 & 144 & 86 \\
\hline CTTCGATGGT & AW791136 & Mannosyl-oligosaccharide $\alpha$-1,2-mannosidase & 55 & 25 & 86 \\
\hline GTGAAATCCG & AW790071 & $\operatorname{gEgh} 7.2$ & 27 & 80 & 84 \\
\hline TCGAAGGGTG & AW788687 & GEL1 protein & 70 & 49 & 75 \\
\hline TGACCACGTT & AW791307 & Novel EST (D00580) & 33 & 48 & 72 \\
\hline CGTGGGGATA & AW791719 & Cytochrome p450-like TBP & 49 & 63 & 70 \\
\hline CGAGCGGTGA & AW791967 & Ubiquitin conjugating enzyme E2-16kD & 53 & 77 & 67 \\
\hline
\end{tabular}

\footnotetext{
${ }^{a}$ Homology is based on expect values of BlastX, BlastN, or tBlastX matches $\mathrm{P}(\mathrm{N}) \leq 1 \mathrm{e}^{-5}$. EST $=$ expressed sequence tag. Number of tags for ungerminated
} conidia (Ug), germinated conidia (Ge), and appressoria (Ap) are given. 
Table 3. Blumeria graminis genes displaying significant changes in transcript abundance during germination and appressorium differentiation ${ }^{\mathrm{a}}$

\begin{tabular}{|c|c|c|c|c|c|c|c|}
\hline Tag sequence ${ }^{\mathrm{b}}$ & $\begin{array}{c}\text { GenBank } \\
\text { accession no. }\end{array}$ & Homology ${ }^{c}$ & $\begin{array}{l}\text { Putative } \\
\text { function }^{d}\end{array}$ & $\mathbf{U g}$ & Ge & Ap & $\mathbf{T P}$ \\
\hline \multicolumn{8}{|l|}{ Increase, both $^{\mathrm{e}}$} \\
\hline \multicolumn{8}{|c|}{ Clones with homologues only in pathogenic fungi } \\
\hline GCACTATTGA & AW788796 & Mycosphaerella graminicola EST AW181057 & $\ldots$ & 56 & 91 & 141 & 1 \\
\hline \multicolumn{8}{|c|}{ Clones with homologues only in fungi } \\
\hline CTCGGCCCAG & AW791162 & 20-kDa subunit V-ATPase (Hex1) & Cell structure & 12 & 44 & 89 & 2 \\
\hline \multicolumn{8}{|c|}{ Clones with homologues in fungi and other organisms } \\
\hline \multirow{2}{*}{\multicolumn{8}{|c|}{$\begin{array}{l}\text { Increase, } \mathbf{G e}^{\mathrm{f}} \\
\text { Clones with homologues only in pathogenic fungi }\end{array}$}} \\
\hline & & & & & & & \\
\hline ACACCAACAA & AW790842 & Infected barley EST BE215687 & & 17 & 34 & 23 & 1 \\
\hline AGTGCACTTA & AW791209 & Retrotransposon & Transposon & 13 & 31 & 23 & 2 \\
\hline CAAGCGAATA* & AW787876 & Magnaporthe grisea EST AI068602 & $\ldots$ & 24 & 45 & 45 & 3 \\
\hline GTGAAATCCG & AW790071 & gEgh7.2 protein & $\ldots$ & 27 & 80 & 84 & 4 \\
\hline \multicolumn{8}{|c|}{ Clones with homologues only in fungi } \\
\hline AAGATGAGTT* $*$ & AW788544 & Aspergillus nidulans EST AA785380 & & 1 & 9 & 6 & 5 \\
\hline GACAGAAATC* & AW788989 & Flavoprotein & Electron trans. & 4 & 14 & 9 & 6 \\
\hline GATAGTCTTA & AW791824 & Ste12 homologue & Transcription & 0 & 7 & 5 & 7 \\
\hline TAAATAGATT & AW791476 & Rasp f 7 & $\ldots$ & 2 & 16 & 7 & 8 \\
\hline TTTCAATCTG & AW790713 & Lipase 2 precursor & Fatty acid met. & 32 & 134 & 135 & 9 \\
\hline \multicolumn{8}{|c|}{ Clones with homologues in fungi and other organisms } \\
\hline AAAAGCGAAA & AW792122 & S-adenosylmethionine synthetase & Amino acid met. & 1 & 12 & 12 & 10 \\
\hline AAGTTGGTTG & AW788464 & 60S ribosomal protein $\mathrm{Rp} 23$ & Protein syn. & 6 & 20 & 22 & 11 \\
\hline AATTATGGGC & AW791241 & Triticum aestivum EST BE402096 & & 7 & 19 & 12 & 12 \\
\hline ACCCCAGAAG* & AW788491 & NADH-ubiquinone oxidoreductase & Electron trans. & 0 & 6 & 2 & 13 \\
\hline ACTTGAGCCT & AW792262 & F1n21.11; similar to glyoxal oxidase & $\ldots$ & 19 & 38 & 47 & 14 \\
\hline ATGCTTCTCA & AW789444 & $40 \mathrm{~S}$ ribosomal protein $\mathrm{S} 12$ & Protein syn. & 3 & 12 & 6 & 15 \\
\hline ATTGCAGCTG* & AW792127 & Pyruvate dehydrogenase E1 component & Glycolysis & 2 & 13 & 9 & 16 \\
\hline CGAGCGGTGA & AW791967 & Ubiquitin conjugating enzyme & Proteolysis & 53 & 77 & 67 & 17 \\
\hline GAGGTCGGGC & AW791580 & Male sterility 2-like protein & Cell structure & 58 & 86 & 112 & 18 \\
\hline GCATCTTTCT & AW790787 & Saccharopine dehydrogenase & Amino acid met. & 6 & 17 & 9 & 19 \\
\hline GTCAGTTTTA* & AW788571 & Glycogen debranching enzyme & Metabolism & 3 & 12 & 10 & 20 \\
\hline TAAAGCCTGC & AW789051 & Ribosomal protein L27 & Protein syn. & 0 & 6 & 5 & 21 \\
\hline TGCTGTCGAA* & AW788777 & Plasma membrane H+-ATPase & Transport & 2 & 11 & 4 & 22 \\
\hline TGTCAGCGGA & AW792301 & 186K protein (CgMMV) & $\ldots$ & 0 & 6 & 14 & 23 \\
\hline TTAAATTGGC & AW788299 & ADP, ATP carrier protein & Transport & 4 & 21 & 14 & 24 \\
\hline TTGGTCTTGG & AW791393 & $40 \mathrm{~S}$ ribosomal protein $\mathrm{S} 4$ & Protein syn. & 4 & 15 & 13 & 25 \\
\hline \multicolumn{8}{|c|}{ Clones with no known homologues } \\
\hline AAGCGAATTA & AW789659 & Novel EST (C01261) & $\ldots$ & 0 & 6 & 2 & 26 \\
\hline ААТССТТСТT & AW790162 & Novel EST (C01556) & $\ldots$ & 7 & 23 & 18 & 27 \\
\hline АСАСТСТТTТ & AW792729 & Novel EST (D01357) & $\ldots$ & 22 & 41 & 35 & 28 \\
\hline CGGAGCTTCC & AW791331 & Novel EST (D00478) & $\ldots$ & 13 & 29 & 45 & 29 \\
\hline CTCGGATTTG & AW791259 & Novel EST (D00555) & $\ldots$ & 5 & 18 & 10 & 30 \\
\hline GAAGTGTAGT & AW790551 & Novel EST (D00034) & $\ldots$ & 8 & 22 & 14 & 31 \\
\hline GGGCGTTATT* & AW789271 & Novel EST (C01025) & $\ldots$ & 0 & 8 & 2 & 32 \\
\hline TGGTTCGTCT & AW792527 & Novel EST (D01245) & $\ldots$ & 0 & 6 & 1 & 33 \\
\hline TTACCTGGCG* & AW790603 & Novel EST (D00063) & $\ldots$ & 9 & 26 & 14 & 34 \\
\hline \multicolumn{8}{|l|}{ Increase, $\mathrm{Ug}$ to $\mathrm{Ap}^{\mathrm{g}}$} \\
\hline \multicolumn{8}{|c|}{ Clones with homologues only in pathogenic fungi } \\
\hline ACCCAGCTTT* & AW788054 & Retrotransposon & Transposon & 0 & 2 & 6 & 1 \\
\hline CAAAATGCAG* & AW792327 & gEgh 16.2 protein & $\ldots$ & 9 & 12 & 24 & 2 \\
\hline \multirow[t]{2}{*}{ GGCCAACTTG* } & AW788630 & Retrotransposon & Transposon & 2 & 5 & 12 & 3 \\
\hline & & & & & \multicolumn{3}{|c|}{ (continued on next page) } \\
\hline
\end{tabular}

$\bar{a}$ Number of tags for ungerminated conidia (Ug), germinated conidia (Ge), and appressoria (Ap) are given. The transcript profile (TP) divides changes in transcriptional abundance into one of 10 profiles as described.

${ }^{\mathrm{b}}$ Tags matching clones lacking a polyA tail are labeled with an asterisk.

${ }^{c}$ Homology is based on expect values of BlastX, BlastN, and tBlastX matches of less than $\mathrm{e}^{-5}$; EST $=$ expressed sequence tag. Differences in transcript levels are significant at the $95 \%(P \leq 0.05)$ confidence interval.

${ }^{\mathrm{d}}$ Electron trans. $=$ electron transport, met. $=$ metabolism, syn. $=$ synthesis, degrad. $=$ degradation, mod. $=$ modifications, $\mathrm{FA}=$ fatty acid, chrom. $=$ chromosome.

${ }^{\mathrm{e}}$ Genes displaying a significant increase in transcript abundance both during germination and during appressorium formation.

${ }^{\mathrm{f}}$ Genes displaying a significant increase in transcript abundance during germination only.

${ }^{\mathrm{g}}$ Genes displaying a significant increase in transcript abundance between ungerminated conidia and appressoria but not attributable to germination or appressorium formation only.

${ }^{\text {h }}$ Genes displaying a significant increase in transcript abundance during appressorium formation only.

${ }^{\mathrm{i}}$ Genes displaying a significant increases in transcript abundance during germination followed by a significant decrease during appressorium formation.

${ }^{\mathrm{j}}$ Genes displaying a significant decrease in transcript abundance during germination followed by a significant increase during appressorium formation.

${ }^{\mathrm{k}}$ Genes displaying a significant decrease in transcript abundance during appressorium formation only.

${ }^{1}$ Genes displaying a significant decrease in transcript abundance between ungerminated conidia and appressoria but not attributable to germination or appressorium formation only.

${ }^{\mathrm{m}}$ Genes displaying a significant decrease in transcript abundance during germination only.

${ }^{\mathrm{n}}$ Genes displaying a significant decrease in transcript abundance both during germination and during appressorium formation. 
Table 3. (continued from preceding page)

\begin{tabular}{|c|c|c|c|c|c|c|c|}
\hline Tag sequence $^{b}$ & $\begin{array}{l}\text { GenBank } \\
\text { accession }\end{array}$ & Homology & $\begin{array}{l}\text { Putative } \\
\text { function }^{d}\end{array}$ & $\mathbf{U g}$ & Ge & Ap & $\mathbf{T P}$ \\
\hline GTAAGATGCA & AW789153 & gEgh16 protein & $\ldots$ & 0 & 4 & 11 & 4 \\
\hline TTACACCCAA* & AW788481 & Acid proteinase Eapc & Protein degrad. & 0 & 3 & 6 & 5 \\
\hline \multicolumn{8}{|c|}{ Clones with homologues only in fungi } \\
\hline GGCGAAGGGT & AW792470 & Hypothetical protein (S. pombe) & $\ldots$ & 2 & 5 & 10 & 6 \\
\hline \multicolumn{8}{|c|}{ Clones with homologues in fungi and other organisms } \\
\hline AGTCAACGAA & AW789369 & Sorghum bicolour EST BE362624 & $\ldots$ & 3 & 6 & 13 & 7 \\
\hline CGGTCCTGGA* & AW790176 & Calnexin & Protein mod. & 0 & 4 & 6 & 8 \\
\hline GAGGTCCCCG* & AW791952 & Pol polyprotein & Transposon & 2 & 7 & 12 & 9 \\
\hline GGCCGGATGG & AW790637 & Serine/threonine protein kinase & Signaling & 5 & 7 & 15 & 10 \\
\hline GGGGCGTGTC & AW790400 & ORF g (Drosophila sp.) & $\ldots$ & 2 & 6 & 10 & 11 \\
\hline \multicolumn{8}{|c|}{ Clones with no known homologues } \\
\hline AAATGCATCG & AW789908 & Novel EST (C01409) & $\ldots$ & 0 & 2 & 9 & 12 \\
\hline \multicolumn{8}{|l|}{ Increase, $\mathrm{Ug}$ to $\mathrm{Ap}^{\mathrm{g}}$} \\
\hline AGATAAGGGG* & AW792557 & Novel EST (D01262) & $\ldots$ & 1 & 3 & 10 & 13 \\
\hline AGCTGGCGCC & AW791652 & Novel EST (D00722) & $\ldots$ & 1 & 2 & 8 & 14 \\
\hline CATCGCGGAC & AW790517 & Novel EST (D00015) & $\ldots$ & 4 & 7 & 15 & 15 \\
\hline CGACAGGGAT & AW789916 & Novel EST (C01414) & $\ldots$ & 0 & 1 & 6 & 16 \\
\hline GCTGCACGCT & AW792218 & Novel EST (D01075) & $\ldots$ & 13 & 19 & 27 & 17 \\
\hline GTGAATGCGT & AW787981 & Novel EST (C00012) & $\ldots$ & 3 & 6 & 12 & 18 \\
\hline TTGGAGCAGG* & AW788004 & Novel EST (C00031) & $\ldots$ & 12 & 19 & 29 & 19 \\
\hline TTGGCTTCAG & AW791104 & Novel EST (D00401) & $\ldots$ & 3 & 8 & 12 & 20 \\
\hline \multicolumn{8}{|l|}{ Increase, Ap only ${ }^{h}$} \\
\hline \multicolumn{8}{|c|}{ Clones with homologues only in pathogenicfungi } \\
\hline AGTGCTCTTC* & AW788879 & Retrotransposon & Transposon & 17 & 18 & 40 & 1 \\
\hline GACCGCTTGG* & AW791069 & Metarrhizium anisopliae EST AJ273279 & $\ldots$ & 14 & 19 & 43 & 2 \\
\hline GGAGTTTGAA* & AW789327 & gEgh16.3 protein & $\ldots$ & 1 & 0 & 8 & 3 \\
\hline \multicolumn{8}{|c|}{ Clones with homologues only in fungi } \\
\hline CGCGCAATGA* & AW788949 & Neurospora crassa EST AI397742 & $\ldots$ & 16 & 27 & 149 & 4 \\
\hline GCTATCCGTG & AW791931 & Aspergillus nidulans EST AA785675 & $\ldots$ & 0 & 0 & 7 & 5 \\
\hline TCGAAGGGTG & AW790942 & GEL 1 & Cell structure & 70 & 49 & 75 & 6 \\
\hline \multicolumn{8}{|c|}{ Clones with homologues in fungi and other organisms } \\
\hline ATGGGTGGAC & AW792426 & Related to chitinase 3 & Cell structure & 52 & 45 & 90 & 7 \\
\hline CAGGTCAGTG & AW791038 & Succinyl-CoA:3-ketoacid-CoA transferase & Lipid/FA met. & 13 & 6 & 25 & 8 \\
\hline СCСАTTTAАC* & AW791418 & Synaptic glycoprotein SC2 spliced variant & $\ldots$ & 1 & 0 & 9 & 9 \\
\hline CCGCAGGGCT & AW790864 & Subtilisin-like serine protease & Protein mod. & 159 & 135 & 212 & 10 \\
\hline CGGACCAAAG* & AW788593 & Histone $\mathrm{H} 3$ & Chrom. structure & 5 & 6 & 21 & 11 \\
\hline GCCGCGGTAT & AW790645 & 3-ketoacyl-CoA thiolase & Fatty acid met. & 5 & 7 & 17 & 12 \\
\hline GCGCGTCAAT* & AW789921 & Triosephosphate isomerase & Glycolysis & 24 & 25 & 47 & 13 \\
\hline GCTAACGCAG & AW791080 & Peptidyl-prolyl cis-trans isomerase & Protein mod. & 158 & 143 & 217 & 14 \\
\hline GGCCTGGCTC** & AW788163 & Serine proteinase Stubble & Protein mod. & 16 & 12 & 25 & 15 \\
\hline TGTGGGACGA & AW788278 & Chitinase & Cell structure & 1 & 3 & 12 & 16 \\
\hline \multicolumn{8}{|c|}{ Clones with no known homologues } \\
\hline AAGTCGATCG & AW788723 & Novel EST (C00658) & $\ldots$ & 5 & 9 & 37 & 17 \\
\hline ACAGGACAAA & AW792757 & Novel EST (D01374) & $\ldots$ & 18 & 27 & 49 & 18 \\
\hline ATCAAGTGAC & AW790594 & Novel EST (D00057) & $\ldots$ & 6 & 14 & 30 & 19 \\
\hline ATCTTGGCCG & AW788016 & Novel EST (C00041) & $\ldots$ & 1 & 0 & 6 & 20 \\
\hline CACAATTTCC & AW792144 & Novel EST (D01030) & $\ldots$ & 0 & 0 & 6 & 21 \\
\hline CCCTCTGGGT* & AW790222 & Novel EST (C01591) & $\ldots$ & 2 & 0 & 7 & 22 \\
\hline CTCGACGGAG & AW789531 & Novel EST (C01180) & $\ldots$ & 9 & 16 & 46 & 23 \\
\hline GAAGCTGGGA & AW790697 & Novel EST (D00133) & $\ldots$ & 11 & 12 & 37 & 24 \\
\hline GCGGCTTGCT & AW789993 & Novel EST (C01455) & $\ldots$ & 2 & 7 & 20 & 25 \\
\hline GCTGGACAGG & AW791982 & Novel EST (D00936) & $\ldots$ & 30 & 30 & 53 & 26 \\
\hline GGATACACTG & AW792383 & Novel EST (D01163) & $\ldots$ & 5 & 9 & 27 & 27 \\
\hline GGCCCATAGG & AW789547 & Novel EST (C01191) & $\ldots$ & 34 & 34 & 91 & 28 \\
\hline GGGATGGGTA & AW792418 & Novel EST (D01183) & $\ldots$ & 0 & 0 & 9 & 29 \\
\hline GTCCGCTTTC** & AW791182 & Novel EST (D00447) & $\ldots$ & 6 & 7 & 18 & 30 \\
\hline TATCGGCGCT & AW791253 & Novel EST (D00552) & $\ldots$ & 31 & 19 & 38 & 31 \\
\hline TGACCACGTT & AW791307 & Novel EST (D00580) & $\ldots$ & 33 & 48 & 72 & 32 \\
\hline TTACTCCACT & AW792226 & Novel EST (D01079) & $\ldots$ & 16 & 26 & 50 & 33 \\
\hline Increase, decrease ${ }^{i}$ & & & & & & & \\
\hline Clones with homolo & es only in path & enic fungi & & & & & \\
\hline TATGAAATTA & AW790659 & Mycosphaerella graminicola EST AW179988 & $\ldots$ & 23 & 40 & 1 & 1 \\
\hline TCCTACATAG* & AW787954 & Retrotransposon & Transposon & 23 & 67 & 32 & 2 \\
\hline TGTCATAGCT & AW790559 & Retrotransposon & Transposon & 95 & 144 & 86 & 3 \\
\hline Clones with homolo & es only in fung & & & & & & \\
\hline TAAAGTTGAT & AW791231 & Sterigmatocystin biosynthesis p450 & & & & & \\
\hline & & monooxygenase & Secondary met. & 6 & 18 & 7 & 4 \\
\hline Clones with homolo & es in fungi and & her organisms & & & & & \\
\hline ACAAAAAAAA & AW788980 & $60 \mathrm{~S}$ ribosomal protein $\mathrm{Y} 16$ & Protein syn. & 7 & 21 & 6 & 5 \\
\hline ACACCTAGAT & AW789656 & Enolase & Glycolysis & 39 & 73 & 13 & 6 \\
\hline AGTGTTACAC** & AW787959 & Bip protein & Protein mod. & 5 & 34 & 15 & 7 \\
\hline & & & & & & ued or & page \\
\hline
\end{tabular}


patterns of mRNA flux (Table 3). Approximately $60 \%$ of these genes have functionally characterized homologues from other organisms, giving an insight into their putative roles in mildew development. As more EST and genome sequences becomes available, it is envisaged that many of the ESTs of unknown function will have identifiable homologues in pathogenic or nonpathogenic filamentous fungi.

It is evident that genes encoding proteins with associated functions tend to display similar fluxes in mRNA content. The genes for several ribosomal proteins fall into classes B, E, and G. Overall, this may imply an increased demand for protein synthesis during germination and a subsequent decline during further development. In Aspergillus nidulans, cloning of genes with increased accumulation of mRNA during conidial germination compared with mycelial growth resulted in the isolation of 10 cDNAs, all of which encoded ribosomal proteins (d'Enfert 1997).

The supply of glycerol and conversion of stored energy during conidial germination and host penetration has been the subject of recent study (Thines et al. 2000). Several catabolic pathways are represented in the SAGE analysis. Increased presence of transcript for a glycogen debranching enzyme (B20, Table 3) during germination is suggestive of the breakdown of glycogen. Three glycolytic enzymes, enolase (E6, Table 3), fructose bisphosphate aldolase (E11, Table 3), and pyruvate kinase (E15, Table 3), display increasing mRNA levels during germination that subsequently fall during appressorium formation. In addition, lower quantities of mRNA for glyceraldehyde 3-phosphate dehydrogenase are detected during appressorium formation (G19, Table 3), whereas one of the com-

Table 3. (continued from preceding page)

\begin{tabular}{|c|c|c|c|c|c|c|c|}
\hline Tag sequence $^{b}$ & $\begin{array}{l}\text { GenBank } \\
\text { accession }\end{array}$ & Homology ${ }^{c}$ & $\begin{array}{l}\text { Putative } \\
\text { function }\end{array}$ & $\mathbf{U g}$ & Ge & Ap & $\mathbf{T P}$ \\
\hline ATGGAAGTAA & AW789651 & Ribosomal protein L14 & Protein syn. & 14 & 28 & 11 & 8 \\
\hline CAATGGAATT & AW790608 & 40s ribosomal protein $\mathrm{S} 17$ & Protein syn. & 9 & 24 & 9 & 9 \\
\hline GCGAACATTG & AW788822 & Peroxisomal hydratase-dehydrogenase-epimerase & Fatty acid met. & 12 & 24 & 10 & 10 \\
\hline GGAAATAGAG & AW790281 & Fructose-bisphosphate aldolase & Glycolysis & 13 & 32 & 12 & 11 \\
\hline GGTTTCGGGC & AW788286 & $60 \mathrm{~S}$ ribosomal protein $\mathrm{P} 2$ & Protein syn. & 46 & 74 & 52 & 12 \\
\hline GTGTAACTAA* & AW787924 & ATP synthase $\gamma$ chain & Electron trans. & 3 & 13 & 2 & 13 \\
\hline TAGTAAATTT & AW789452 & Mannosyl-oligosaccharide $\alpha$-1,2-mannosidase & Protein mod. & 10 & 23 & 3 & 14 \\
\hline TCATTTTTCA & AW791226 & Pyruvate kinase & Glycolysis & 11 & 34 & 3 & 15 \\
\hline TTCTGGTACA* & AW789493 & Protein disulphide isomerase & Protein mod. & 9 & 23 & 5 & 16 \\
\hline \multicolumn{8}{|c|}{ Clones with no known homologues } \\
\hline AAATAAAAAC* & AW789299 & Novel EST (C01043) & $\ldots$ & 11 & 23 & 7 & 17 \\
\hline AACTTGAATT & AW790156 & Novel EST (C01553) & $\ldots$ & 1 & 8 & 0 & 18 \\
\hline ATATAGGCGG & AW791030 & Novel EST (D00348) & $\ldots$ & 10 & 50 & 31 & 19 \\
\hline CAGAGAGGCA* & AW789769 & Novel EST (C01328) & $\ldots$ & 44 & 85 & 41 & 20 \\
\hline \multicolumn{8}{|l|}{ Decrease, increase $\mathrm{j}^{\mathrm{j}}$} \\
\hline \multicolumn{8}{|c|}{ Clones with homologues only in fungi } \\
\hline AAATACCCTG & AW791784 & Cellulose growth specific protein & Host cell degrad. & 32 & 16 & 38 & 1 \\
\hline AAGGGAGGAT & AW791711 & Metallothionein & $\ldots$ & 286 & 209 & 501 & 2 \\
\hline ACCAAACTCA & AW790393 & Ribosomal protein S28 & Protein syn. & 8 & 1 & 13 & 3 \\
\hline CCAACGTTTG & AW790498 & Thioredoxin & Redox reactions & 41 & 21 & 38 & 4 \\
\hline CTTCGATGGT* & AW791136 & Mannosyl-oligosaccharide $\alpha$-1,2-mannosidase & Protein mod. & 55 & 25 & 86 & 5 \\
\hline \multicolumn{8}{|l|}{ Decrease, increase $\mathrm{j}^{\mathrm{j}}$} \\
\hline GAGCGCGTGA & AW790920 & Isotrichodermin C-15 hydroxylase & Other met. & 49 & 13 & 36 & 6 \\
\hline GTTCAAGTTG* & AW790846 & Peroxisomal membrane protein pmp $20 p$ & Detoxification & 21 & 6 & 25 & 7 \\
\hline TATCGCGGTC* & AW789111 & Phosphatidylserine synthase & Cell structure & 8 & 1 & 8 & 8 \\
\hline \multicolumn{8}{|c|}{ Clones with no known homologues } \\
\hline TCACCCCACT & AW791647 & Novel EST (D00719) & $\ldots$ & 156 & 88 & 129 & 9 \\
\hline TCCACTGGAT & AW791134 & Novel EST (D00419) & $\ldots$ & 46 & 28 & 53 & 10 \\
\hline \multicolumn{8}{|l|}{ Decrease, Ap onlyk } \\
\hline \multicolumn{8}{|c|}{ Clones with homologues only in fungi } \\
\hline AAGAGAATTT & AW788489 & Hypothetical protein $(S$. pombe) & $\ldots$ & 2 & 6 & 0 & 1 \\
\hline CGAAAAAAAA & AW791335 & Neurospora crassa EST AA901986 & $\ldots$ & 9 & 12 & 2 & 2 \\
\hline GAAAAAAAAA & AW789324 & Aut1 & Transport & 6 & 13 & 3 & 3 \\
\hline GATAAAAAGT* & AW792656 & Neurospora crassa EST AW712490 & $\ldots$ & 30 & 30 & 10 & 4 \\
\hline GATCCTAGCT & AW790464 & Hypothetical protein $(S$. pombe $)$ & $\ldots$ & 3 & 9 & 1 & 5 \\
\hline GTTCAATTTT* & AW789202 & NADH-ubiquinone oxidoreductase & Electron trans. & 1 & 6 & 0 & 6 \\
\hline TAAAACCTCT & AW792722 & Tropomyosin & Cytoskeleton & 5 & 13 & 3 & 7 \\
\hline TACTACATCT & AW790651 & QDE2-gene silencing & Transcription & 3 & 7 & 0 & 8 \\
\hline \multicolumn{8}{|c|}{ Clones with homologues in fungi and other organisms } \\
\hline AAGGAAAGCG & AW790531 & $14-3-3$ protein & Transcription & 10 & 16 & 5 & 9 \\
\hline AATAGATGAA* & AW790370 & Cyclophilin & Protein mod. & 5 & 8 & 1 & 10 \\
\hline AATTTCAAAT & AW788912 & Nucleoside diphosphate kinase & Metabolism & 13 & 23 & 6 & 11 \\
\hline ACGCATTAAA & AW792002 & Profilin A & Cytoskeleton & 7 & 16 & 6 & 12 \\
\hline AGGTATAGTT & AW791459 & Pyruvate carboxylase & Gluconeogenesis & 18 & 29 & 9 & 13 \\
\hline ATATCGAACT & AW787872 & 60S ribosomal protein L22 & Protein syn. & 9 & 19 & 4 & 14 \\
\hline CAAAAAAAAA & AW791903 & GTP-binding nuclear protein GSP1/CNR1 & Protein mod. & 25 & 31 & 10 & 15 \\
\hline CACAGGCGTT & AW790930 & GABA-specific permease & Transport & 2 & 9 & 1 & 16 \\
\hline \multirow[t]{2}{*}{ GACTCAATCT } & AW792466 & Serine hydroxymethyltransferase/ ABC & Amino acid met. & & & & \\
\hline & & transporter & /detoxification & 11 & 20 & 6 & 17 \\
\hline GATTGAAAAT** & AW789551 & Catalase/peroxidase & Detoxification & 9 & 15 & 2 & 18 \\
\hline GTACGATAAT & AW788939 & Glyceraldehyde 3-phosphate dehydrogenase & Glycolysis & 30 & 39 & 23 & 19 \\
\hline GTCCCTTACA & AW792607 & Carboxypeptidase Y precursor & Protein mod. & 18 & 29 & 11 & 20 \\
\hline \multirow[t]{2}{*}{ TAGCTCATTG } & AW790216 & Myosin 2 & Cytoskeleton & 2 & 8 & 1 & 21 \\
\hline & & & & & \multicolumn{3}{|c|}{ (continued on next page) } \\
\hline
\end{tabular}


ponents of pyruvate dehydrogenase (B16, Table 3) displays an increase in transcript level during germination only. Together, the observed changes are indicative of an active participation of glycolysis during germination that is subsequently downregulated during appressorium formation. An exception, the gene for triosephosphate isomerase (D13, Table 3), exhibits higher transcript abundance during appressorium differentiation only. This may reflect shunting of glycolytic intermediates to membrane synthesis or possibly glycerol formation suggested to be associated with the increased turgor pressure observed in the appressoria of M. grisea (de Jong et al. 1997). Conversely, decreasing transcript levels for two genes encoding enzymes associated with gluconeogenesis, pyruvate carboxylase (G13, Table 3) and fructose-1,6-bisphosphatase (H5, Table 3), during appressorial development is apparent. Transcripts of a lipase precursor (B9, Table 3) accumulate during germination and appressorium formation, whereas two $\beta$-oxidation enzymes, succinyl CoA:3-ketoacid transferase (D8, Table 3) and 3-ketoacyl-CoA thiolase (D12, Table 3), show increases in mRNA content during the appressorial development phase only. Interestingly, succinyl CoA:3-ketoacid transferase is associated with ketone body catabolism. This suggests a possible active utilization of lipids in preference to carbohydrates in the later stages of germling development.

The Hexl gene of Neurospora crassa recently has been identified as a core component of the Woronin body (Jedd and Chua 2000). Woronin bodies have been observed in many filamentous fungi, including B. graminis (McKeen 1971), and are thought to act in plugging septal pores after damage to hyphae in order to prevent uncontrolled leakage of the cytoplasm (Jedd and Chua 2000). The increased presence of transcripts of the $B$. graminis Hexl homologue observed during germination

Table 3. (continued from preceding page)

\begin{tabular}{|c|c|c|c|c|c|c|c|}
\hline Tag sequence ${ }^{b}$ & $\begin{array}{l}\text { GenBank } \\
\text { accession }\end{array}$ & Homology $y^{c}$ & $\begin{array}{l}\text { Putative } \\
\text { functiond }\end{array}$ & Ug & Ge & Ap & $\mathbf{T P}$ \\
\hline TATTTTGTAT & AW791728 & Bleomycin hydrolase & Detoxification & 1 & 7 & 0 & 22 \\
\hline TCGTAAAGGT & AW792640 & Rho1 & Signaling & 18 & 17 & 6 & 23 \\
\hline TTGGCTCAAT & AW789848 & 40s ribosomal protein S5 & Protein syn. & 12 & 20 & 5 & 24 \\
\hline \multicolumn{8}{|c|}{ Clones with no known homologues } \\
\hline ACACATTTTT** & AW789718 & Novel EST (C01294) & $\ldots$ & 8 & 12 & 3 & 25 \\
\hline ATGATTTTCT & AW791805 & Novel EST (D00816) & $\ldots$ & 3 & 7 & 0 & 26 \\
\hline АTTTTTTCTC & AW791656 & Novel EST (D00724) & $\ldots$ & 3 & 7 & 0 & 27 \\
\hline GTAGAAAAAG & AW791721 & Novel EST (D00765) & $\ldots$ & 5 & 14 & 2 & 28 \\
\hline TAAAAAAAAA & AW791319 & Novel EST (D00586) & $\ldots$ & 10 & 10 & 2 & 29 \\
\hline TATTGGAAGT** & AW789289 & Novel EST (C01036) & $\ldots$ & 2 & 8 & 1 & 30 \\
\hline \multicolumn{8}{|l|}{ Decrease, $\mathrm{Ug}$ to $A p^{1}$} \\
\hline \multicolumn{8}{|c|}{ Clones with homologues only in pathogenic fungi } \\
\hline ATATAATGTG & AW791976 & Cap20 (Colletotrichum gloeosporioides) & $\ldots$ & 17 & 9 & 6 & 1 \\
\hline \multicolumn{8}{|c|}{ Clones with homologues only in fungi } \\
\hline CATTGAGGAA* & AW789717 & Aspergillus niger EST BE759613 & $\ldots$ & 21 & 17 & 10 & 2 \\
\hline CCAAAAGCAT* & AW788721 & NADH dehydrogenase & Electron trans. & 7 & 5 & 0 & 3 \\
\hline \multicolumn{8}{|c|}{ Clones with homologues in fungi and other organisms } \\
\hline AGGCAAACAG* & AW790435 & Peptidyl-prolyl isomerase & Protein mod. & 8 & 2 & 1 & 4 \\
\hline GTAAAGTTCA & AW792329 & Fructose-1,6-bisphosphatase & Gluconeogenesis & 15 & 11 & 3 & 5 \\
\hline GTTCTTGAGA & AW790515 & МepB & Protein mod. & 14 & 10 & 4 & 6 \\
\hline TATGCGCTCA & AW792070 & Vacuolar ATP synthase subunit E & Transport & 9 & 3 & 1 & 7 \\
\hline \multicolumn{8}{|c|}{ Clones with no known homologues } \\
\hline ACAAAAAAAC & AW791325 & Novel EST (D00475) & $\ldots$ & 10 & 5 & 2 & 8 \\
\hline GAAAGAAGAA & AW791461 & Novel EST (D00604) & $\ldots$ & 6 & 1 & 0 & 9 \\
\hline \multicolumn{8}{|l|}{ Decrease, Ge only ${ }^{\mathrm{m}}$} \\
\hline \multicolumn{8}{|c|}{ Clones with homologues only in fungi } \\
\hline ACAACCTGTA & AW791156 & Aspergillus nidulans EST AI210287 & $\ldots$ & 26 & 8 & 7 & 1 \\
\hline CGCTGTAAAA & AW792085 & Neurospora crassa EST AI319045 & $\ldots$ & 14 & 2 & 1 & 2 \\
\hline GAAGAATTAC & AW792399 & Neurospora crassa EST AA898852 & $\ldots$ & 17 & 3 & 6 & 3 \\
\hline GGCAATTGCT & AW792032 & Neurospora crassa EST AA898854 & $\ldots$ & 6 & 0 & 3 & 4 \\
\hline GGGTTGCAAA* & AW792221 & Aspergillus nidulans EST AI211830 & $\ldots$ & 51 & 15 & 16 & 5 \\
\hline \multicolumn{8}{|c|}{ Clones with homologues in fungi and other organisms } \\
\hline AATGAAGATG & AW791855 & Spore wall maturation protein DIT1 & Cell structure & 11 & 1 & 1 & 6 \\
\hline ACCATTAGGA & AW791200 & Heat shock protein Hsp1 & Stress response & 16 & 3 & 10 & 7 \\
\hline ATACCAAAAT & AW791955 & 30-kDa heat shock protein & Stress response & 63 & 10 & 3 & 8 \\
\hline ATACCGAAAG* & AW790177 & 30-kDa heat shock protein & Stress response & 20 & 5 & 2 & 9 \\
\hline CCCCAACAGA & AW792024 & Type III alcohol dehydrogenase & Other met. & 34 & 9 & 16 & 10 \\
\hline GACAGAATCA* & AW792532 & Ice plant EST AW053761 & $\ldots$ & 37 & 4 & 6 & 11 \\
\hline GCTGCCGCGG* & AW791646 & EFA27 for EF hand & Transcription & 18 & 0 & 1 & 12 \\
\hline TCTACTGGCG & AW790429 & $\alpha$ hemolysin & & 10 & 2 & 0 & 13 \\
\hline TGATAAAGCG & AW790588 & D-xylose reductase II, III & Metabolism & 29 & 12 & 18 & 14 \\
\hline TTGTACGCGA & AW792507 & Unknown protein Homo sapiens & $\ldots$ & 10 & 1 & 2 & 15 \\
\hline \multicolumn{8}{|c|}{ Clones with no known homologues } \\
\hline AAGGGTGTAA & AW792009 & Novel EST (D00951) & $\ldots$ & 36 & 10 & 17 & 16 \\
\hline ATAGACCGTT & AW792043 & Novel EST (D00972) & $\ldots$ & 19 & 7 & 9 & 17 \\
\hline ATATATCAAA & AW791629 & Novel EST (D00709) & $\ldots$ & 9 & 0 & 0 & 18 \\
\hline ATCGGCGTAT** & AW789601 & Novel EST (C01224) & $\ldots$ & 6 & 0 & 2 & 19 \\
\hline ATTAATGCGG & AW792338 & Novel EST (D01140) & $\ldots$ & 40 & 22 & 19 & 20 \\
\hline TCTGTGCAAA & AW791483 & Novel EST (D00617) & $\ldots$ & 9 & 1 & 4 & 21 \\
\hline TTAGTCTGTT & AW792295 & Novel EST (D01117) & $\ldots$ & 55 & 16 & 14 & 22 \\
\hline TTGATTACTC* & AW788433 & Novel EST (C00443) & $\ldots$ & 13 & 4 & 5 & 23 \\
\hline \multicolumn{8}{|l|}{ Decrease bothn } \\
\hline \multicolumn{8}{|c|}{ Clones with homologues only in fungi } \\
\hline AATTAGCTAA & AW791518 & Glucose-repressible gene & Signaling & 113 & 70 & 46 & 1 \\
\hline
\end{tabular}


and appressorium formation (A2, Table 3 ) is consistent with a putative role of Woronin bodies in blocking the septal pore between the conidium and the appressorium, allowing the accumulation of sufficient appressorial turgor pressure required for breaching of the host cell wall. The observation of a Hexl homologue in M. grisea (Jedd and Chua 2000), where host penetration results from massive turgor generation in the appressorium (Howard et al. 1991), supports this theory.

Transcripts of a Myb-like transcription factor are identified with an increasing frequency during both germination and appressorium formation (A3, Table 3 ). It would be of interest to determine if the transcription factor is under the control of the cAMP pathway, which has been implicated in the differentiation of appressoria in many pathogenic fungi, including $B$. graminis (Hall et al. 1999; Kinane et al. 2000).

Gene families are a feature of the results and are well suited to analysis by SAGE because the technique relies on the isolation of a tag from the three prime end of the messenger, a region where most members of gene families display the highest degree of divergence. The $g E g h 16$ gene family has already been mentioned. Six peptidyl-prolyl cis-trans isomerase and cyclophilin genes also have been identified. Three of these genes display significant changes in transcript abundance and, interestingly, the profiles are complementary; the increased accumulation of transcripts of one gene during appressorium formation (D14, Table 3) being shadowed by a decreased presence of the other two (G10 and H4, Table 3). In comparison, the various retroelement gene families predominantly display significant increases in mRNA quantities during germination and appressorium formation.

During early development, the fungal germling must degrade the spore wall and synthesize new cell wall constituents to allow hyphal growth and appressorium differentiation. Therefore, it is to be expected that transcripts of two chitinase homologues became significantly more prevalent as development continued (D7 and D16, Table 3). The relatively high transcript abundance of a homologue of GEL1 (D6, Table 3), required for elongation of 1,3- $\beta$-glucan in Aspergillus fumigatus (Mouyna et al. 2000), is suggestive of the importance of $1,3-\beta$-glucan synthesis in cell wall formation during early fungal development.

Currently available at the NCBI dbEST database are 24,626 sequences from $N$. crassa, 12,993 sequences from $A$. nidulans, and 1,693 sequences from Metarhizium anisopliae; as well as 1,158 sequences from Mycosphaerlla graminicola, 2,681 sequences from $M$. grisea, and 600 sequences from Cladosporium fulvum. In addition, numerous genes from human pathogenic fungi are available for comparison, as well as the complete yeast genome and SAGE expression profiles for 4,665 yeast genes during cell cycle progression in yeast (Velculescu et al. 1997). There exists the opportunity to extend both gene identification and the transcript profiling to related fungi with the aim of building a global relational database comprising functional and expression data for a range of pathogens. Such a database would allow the identification of potential targets of future studies of the genes involved in filamentous fungal growth and pathogenicity.

\section{MATERIALS AND METHODS}

\section{Fungal techniques.}

All experiments were conducted on Blumeria graminis f. sp. hordei race $\mathrm{C} 15$. Maintenance of the fungus on the susceptible barley cv. Golden Promise has been described previously (Kinane et al. 2000). Isolation of the fungal material at different stages of development was achieved by inducing germination and appressorium formation on cellulose membranes as previously described (Kinane et al. 2000). The mean percentage of conidia forming primary germ tubes or appressoria on artificial surfaces was calculated by microscopic evaluation. The SAGE profile for germinating conidia was constructed using RNA from conidia $4 \mathrm{~h}$ after application to cellulose membrane, at which point $82 \%$ had a primary germ tube. This is near the maximum degree of germination observed on either cellulose membranes or barley epidermis (Kinane et al. 2000) and, although slightly higher rates of germination can be established at later time points, $4 \mathrm{~h}$ was deemed appropriate because, at this stage, appressorial germ tube formation is negligible, so that the material is solely indicative of germination. The appressorial SAGE profile was constructed with RNA from conidia after $16 \mathrm{~h}$ of contact with cellulose membrane. At this point, $90 \%$ of the conidia had a primary germ tube, of which $77 \%$ also had swollen appressorial germ tubes and $66 \%$ had fully differentiated appressoria. Again, at this time, the percentage of appressorial germ tube emergence and differentiation was approaching the maximum observed on both cellulose membranes and barley epidermis (Kinane et al. 2000).

\section{RNA techniques.}

Total RNA was isolated for all developmental stages studied following the procedure of Chomczynski and Sacchi (1987) with the addition of $50 \mathrm{mM}$ ascorbic acid to solution D to prevent polyphenol oxidation. PolyA ${ }^{+}$RNA was purified on oligo(dT)-cellulose spin columns (Amersham Pharmacia Biotech) following the manufacturer's specifications. The purity and quality of both total RNA and mRNA preparations was confirmed by denaturing gel electrophoresis (data not shown).

\section{Generation of SAGE profiles.}

For each developmental stage, 2.5 to $5 \mu \mathrm{g}$ of polyA ${ }^{+}$RNA was used to construct SAGE tag populations following the original protocol (Velculescu et al. 1995) and incorporating published improvements (Kenzelmann and Mühlemann 1999). Tag concatamers were cloned into pZero (Invitrogen) and the constructs introduced into Escherichia coli Top10 cells (Invitrogen). Recombinant plasmids were isolated from single colonies employing a Qiagen minikit and sequenced using BigDye terminator technology (Applied Biosystems, Foster City, CA, U.S.A.).

\section{Handling of sequence data.}

Raw sequence files were imported into the DNATools sequence handling program and ambiguous base calls were corrected manually when possible. Following sequence editing, ditags (Velculescu et al. 1995) were extracted using both the automatic extraction facilities within DNATools and a second software package, eSAGE (Margulies and Innis 2000). Both approaches yield a display of individual tags and their frequency of occurrence, and discrepancies between the outputs were resolved manually. Duplicate ditags arising from PCR bias as well as tags originating from the linker sequences automatically were removed from the analyses by the software employed. Tags sequenced only once across the three populations also were removed from the analysis according to Lash and associates (2000) to prevent overestimation of the number of different tags introduced by properties inherent to the SAGE procedure.

\section{Statistical analysis of SAGE data.}

Comparison of SAGE profiles between the three populations studied was accomplished by graphical display using the SAGE utilities available in the DNATools package as well as the statistical package within eSAGE (Margulies and Innis 2000). Statistical analysis using DNATools was performed by comparing the number of tags in two stages using the $\mathrm{Z}$ test as previously described (Altman 1991; Kal et al. 1999). The 
eSAGE software employs the test statistic developed by Audic and Claverie (1997). To avoid erroneous analysis of differences in observed numbers of tags between populations when the number of observations was low (i.e., less than six tags in one population), the statistical significance of observed differences was calculated using the more conservative Fisher Exact test running on the SPSS statistical package (ver. 10 for Windows; SPSS, Chicago).

Statistical analysis of observed differences in tag number were made between ungerminated and germinating conidia, between germinating conidia and appressoria, and, when appropriate, between ungerminated conidia and appressoria. Applying these tests of statistical significance allowed the grouping of genes into several different classes, as follows. (i) A significant increase in transcript abundance was observed during germination (between ungerminated and germinated conidia) as well as during appressorium formation (between germinated conidia and appressoria). (ii) A significant increase in transcript abundance was observed during germination only. (iii) A significant increase in transcript abundance was not detectable between ungerminated and germinated conidia or between germinated conidia and appressoria, but was apparent between ungerminated conidia and appressoria. (iv) A significant increase in transcript abundance was detectable only during appressoria formation. (v) A significant increase in transcript abundance was apparent during germination, followed by a significant decrease during appressorium formation. (vi) A significant decrease in transcript abundance was apparent during germination and was followed by a significant increase in transcript abundance during appressorium formation. (vii) A significant decrease in transcript abundance was observed during appressorium formation only. (viii) A significant decrease in transcript abundance was not detectable between ungerminated and germinated conidia or between germinated conidia and appressoria, but was apparent between ungerminated conidia and appressoria. (ix) A significant decrease in transcript abundance was observed during germination only. ( $\mathrm{x}$ ) A significant decrease in transcript abundance was observed during germination as well as during appressorium formation.

\section{Mapping of SAGE tags.}

Prior to mapping to EST clones, tags were analyzed against the published ribosomal RNA sequences of $B$. graminis $\mathrm{f}$. $\mathrm{sp}$. hordei (Mori et al. 2000; Paulin and Harrington 2000; Saenz and Taylor 1999). Tags present in the ribosomal RNA sequences were regarded as having arisen due to mispriming of residual ribosomal RNA and were discarded from the analysis.

Identification of genes represented by SAGE tags was accomplished using DNATools following principles devised by Velculescu and associates (1995). Briefly, the entire complement of EST sequences from $B$. graminis initially was examined for the presence of a polyA tail. For those sequences containing a polyA tail, the closest NlaIII site was identified and the tag sequences extracted. The extracted tags were labeled "A" to indicate that they arose from a polyA-containing EST. For clones where both the $5^{\prime}$ and $3^{\prime}$ sequence lacked a polyA tail, the most $3^{\prime}$ NlaIII sites in both sequences were identified and the tag immediately adjacent to the $3^{\prime}$ end of the NlaIII recognition site on both DNA strands was used for identification. Such clones, lacking a polyA tail, could yield a maximum of four tags, of which only one would represent the gene in question; therefore, the tags were labeled " $\mathrm{W}$ " or " $\mathrm{C}$ " to indicate from which strand the tag arose. Tags originating from the $5^{\prime}$ and $3^{\prime}$ sequences of such clones were used for mapping only in cases where the correct orientation of the gene could be verified by the alignment of the EST sequence to a homologous sequence (BlastX $\mathrm{P}(\mathrm{N}) \leq 1 \mathrm{e}^{-5}$ ) retrieved from GenBank.
A list of EST clones and their extracted tags was used for mapping of the SAGE tags generated in the course of this project. A complete list of the tags generated by this project, as well as the annotated EST clones to which they were mapped, is available online.

\section{ACKNOWLEDGMENTS}

This article is dedicated to the memory of Jean Sage, who passed away shortly after retiring from research activities. We thank Q. Abdali, B. Anderson, and J. Sage for technical assistance in template preparation and sequencing; and M. Kielland-Brandt for critically reading the manuscript.

\section{LITERATURE CITED}

Altman, D. G. 1991. Pages 162-163 in: Practical Statistics for Medical Research. Chapman-Hall, London.

Altschul, S. F., Gish, W., Miller, W., Myers, E. W., and Lipman, D. J. 1990. Basic local alignment search tool. J. Mol. Biol. 215:403-410.

Altschul, S. F., Madden, T. L., Schäffer, A. A., Zhang, J., Zhang, Z., Miller, W., and Lipman, D. J. 1997. Gapped BLAST and PSI-BLAST: a New generation of protein database search programs. Nucleic Acids Res. 25:3389-3402.

Andrews, G. K. 2000. Regulation of metallothionein gene expression by oxidative stress and metal ions. Biochem. Pharmacol. 59:95-104.

Audic, S., and Claverie, J.-M. 1997. The significance of digital gene expression profiles. Genome Res. 7:986-995.

Chomczynski, P., and Sacchi, N. 1987. Single-step method of RNA isolation by acid guanidium thiocyanate-phenol-chloroform extraction Anal. Biochem. 162:156-159.

Dean, R. A. 1997. Signal pathways and appressorium morphogenesis. Annu. Rev. Phytopathol 35:211-234.

de Jong, J. C., McCormack, B. J., Smirnoff, N., and Talbot, N. J. 1997. Glycerol generates turgor in rice blast. Nature 389:244-245.

d'Enfert, C. 1997. Fungal spore germination: Insights from the molecular genetics of Aspergillus nidulans and Neurospora crassa. Fungal Genet. Biol. 21:163-172.

Eckert, S., Both, M., DeBruijn, A., Mueller, E., and Spanu, P. 2001 Sequence analysis and expression studies of Erysiphe graminis cDNAs from epiphytic mycelium. Fungal Genet. Newsl. 48 (Suppl.):95.

Hall A. A., Bindslev L., Rouster J., Rasmussen S. W., Oliver, R. P., and Gurr, S. J. 1999. Involvement of cAMP and protein kinase A in conidial differentiation by Erysiphe graminis f. sp. hordei. Mol. Plant-Microbe Interact. 12:960-968

Hamer, J. E., and Talbot, N. J. 1998. Infection-related development in the rice blast fungus Magnaporthe grisea. Curr. Opin. Microbiol. 1:693697.

Howard R. J., Ferarri M. A., Roach D. H., and Money N. P. 1991. Penetration of hard surfaces by a fungus employing enormous turgor pressures. Proc. Natl. Acad. Sci. U.S.A. 88:11281-11284.

Howard, R. J., and Valent, B. 1996. Breaking and entering: Host penetration by the fungal rice blast pathogen Magnaporthe grisea. Annu. Rev. Microbiol. 50:491-512.

Hwang, C, and Kolattukudy, P. E. 1995. Isolation and characterization of genes expressed uniquely during appressorium formation by Colletotrichum gloeosporioides conidia induced by the host surface wax. Mol. Gen. Genet. 247:282-294.

Ishii, M., Hashimoto, S., Tsutsumi, S., Wada, Y., Matsushima, K., Kodama, T., and Aburatani, H. 2000. Direct comparison of genechip and SAGE on the quantitative accuracy in transcript profiling analysis. Genomics 68:136-143.

Jedd, G., and Chua, N. 2000. A new self-assembled peroxisomal vesicle required for efficient resealing of the plasma membrane. Nat. Cell Biol. 2:226-231.

Justesen A., Somerville, S., Christiansen, S., and Giese, H. 1996. Isolation and characterization of two novel genes expressed in germinating conidia of the obligate biotroph Erysiphe graminis f. sp. hordei. Gene 170:131-135.

Kal, A. J., van Zonneveld, A. J., Benes, V., van den Berg, M., Groot Koerkamp, M., Albermann, K., Strack, N., Ruitjer, J. M., Richter, A., Dujon, B., Ansorge, W., and Tabak, H. F. 1999. Dynamics of gene expression revealed by comparison of serial analysis of gene expression transcript profiles from yeast grown on two different carbon sources. Mol. Biol. Cell 10:1859-1872.

Kamakura T., Xiao, J.-Z., Choi, W.-B., Kochi, T., Yamaguchi, S., Teraoka, T., and Yamaguchi, I. 1999. cDNA subtractive cloning of genes expressed during early stage of appressorium formation by Magnaporthe grisea. Biosci. Biotechnol. Biochem. 63:1407-1413. 
Kenzelmann, M., and Mühlemann, K. 1999. Substantially enhanced cloning efficiency of SAGE (Serial analysis of gene expression) by adding a heating step to the original protocol. Nucleic Acids Res. 27:917-918.

Kinane, J., Dalvin, S., Bindslev, L., Hall, A., Gurr, S., and Oliver, R. 2000. Evidence that the cAMP pathway controls emergence of both primary and appressorial germ tubes of barley powdery mildew. Mol. PlantMicrobe Interact. 13:494-502.

Kobayashi, I., Tanaka, C., Yamaoka, N., and Kunoh, H. 1991. Morphogenesis of Erysiphe graminis conidia on artificial membranes. Trans. Mycol. Soc. Jpn. 32:187-198.

Kupfer, D. M., Reece, C. A., Clifton, S. W., Roe, B. A., and Prade, R. A 1997. Multicellular ascomycetous fungal genomes contain more than 8000 genes. Fungal Genet. Biol. 21:364-372.

Lash, A. E., Tolstoshev, C. M., Wagner, L., Schuler, G. D., Strausberg, R. L., Riggins, G. J., and Altschul, S. F. 2000. SAGEmap: A public gene expression resource. Genome Res. 10:1051-1060.

Margulies, E. H., and Innis, J. W. 2000. eSAGE: Managing and analyzing data generated with Serial Analysis of Gene Expression (SAGE). Bioinformatics 16:650-651.

McCafferty, H. R. K., and Talbot, N. J. 1998. Identification of three ubiquitin genes of the rice blast fungus Magnaporthe grisea, one of which is highly expressed during initial stages of plant colonization. Curr. Genet. 33:352-361

McKeen, W. E. 1971. Woronin bodies in Erysiphe graminis DC. Can. J. Microbiol. 17:1557-1560.

Mengden, K., Hahn, M., and Deising, H. 1996. Morphogenesis and mechanisms of penetration by plant pathogenic fungi. Annu. Rev. Phytopathol. 34:367-386.

Mori, Y., Sato, Y., and Takamatsu, S. 2000. Molecular phylogeny and radiation time of Erysiphales inferred from the nuclear ribosomal DNA sequences. Mycoscience 41:437-447.

Mouyna, I., Fontaine, T., Vai, M., Monod, M., Fonzi, W. A., Diaquin, M., Popolo, L., Hartland, R. P., and Latge, J.-P. 2000. Glycosylphosphatidylinositol-anchored glucanosyltransferases play an active role in the biosynthesis of the fungal cell wall. J. Biol. Chem. 275:14882-14889.

Paulin, A. E., and Harrington, T. C. 2000. Phylogenetic placement of ana- morphic species of Chalara among Ceratocystis species and other ascomycetes. Stud. Mycol. 45:209-222.

Polyak, K., Xia, Y., Zweier, J. L., Kinzler, K. W., and Vogelstein, B. 1997. A model for p53-induced apoptosis. Nature 389:300-305.

Saenz, G. S., and Taylor, J. W. 1999. Phylogeny of the Erysiphales (powdery mildews) inferred from internal transcribed spacer ribosomal DNA sequences. Can. J. Bot. 77:150-168.

Schena, M., Shalon, D., Davis, R. W., and Brown, P. O. 1995. Quantitative monitoring of gene expression patterns with a complimentary DNA microarray. Science 270:467-470.

Thines, E., Weber, R. W. S., and Talbot, N. J. 2000. MAP kinase and protein kinase A-dependent mobilization of triacylglycerol and glycogen during appressorium turgor generation by Magnaporthe grisea. Plant Cell 12:1703-1718.

Thomas, S. W., Rasmussen, S. W., Glaring, M. A., Rouster, J. A., Christiansen, S. K., and Oliver, R. P. 2001. Gene identification in the obligate fungal pathogen Blumeria graminis by expressed sequence tag analysis. Fungal Genet. Biol. 33:195-211.

Velculescu, V. E., Zhang, L., Zhou, W., Vogelstein, J., Basrai, M. A., Bassett, D. E., Jr., Hieter, P., Vogelstein, B., and Kinzler, K. W. 1997. Characterization of the yeast transcriptome. Cell 88:243-251.

Velculescu, V. E., Zhang, L., Vogelstein, B., and Kinzler, K. W. 1995. Serial analysis of gene expression. Science 270:484-487.

$\mathrm{Xu}$, J.-R. 2000. MAP kinases in fungal pathogens. Fungal Genet. Biol. 31:137-152.

Yu, J., Zhang, L., Hwang, P. M., Rago, C., Kinzler, K. W. and Vogelstein, B. 1999 Identification and classification of p53-regulated genes. Proc. Natl. Acad. Sci. U.S.A. 96:14517-14522.

Zhang, Z., Hall, A., Perfect, E., and Gurr, S. J. 2000. Differential expression of two Blumeria graminis chitin synthase genes. Mol. Plant Pathol. $1: 125-138$.

\section{AUTHOR-RECOMMENDED INTERNET RESOURCES}

DNATools sequence handling program: www.dnatools.dk

Complete list of tags generated by this project: www.crc.dk/phys/blumeria 